\title{
Outcomes of Living and Deceased Donor Liver Transplant Recipients With Hepatocellular Carcinoma: Results of the A2ALL Cohort ${ }^{\dagger}$
}

\author{
L. M. Kulik a , R. A. Fisher, *, D. R. Rodrigoc, \\ R. S. Brown, Jr. ${ }^{\text {d }}$, C. E. Freise ${ }^{\text {, }}$, A. Shaked ${ }^{\text {, }}$ \\ J. E. Everhart' ${ }^{g}$, G. T. Everson ${ }^{h}$, J. C. Hongi, \\ P. H. Hayashi' , C. L. Berg' ${ }^{k}$, A. S. F. Lok' \\ and the A2ALL Study Group
}

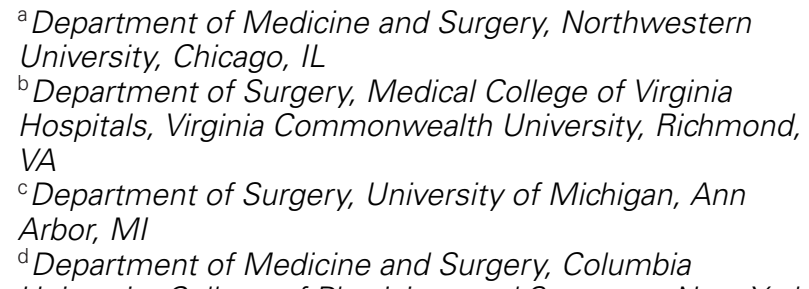
University College of Physicians and Surgeons, New York, NY

e Department of Surgery, University of California, San

Francisco, San Francisco, CA

${ }^{\mathrm{f}}$ Department of Surgery, University of Pennsylvania,

Philadelphia, PA

'Department of Medicine, University of Colorado, Denver, Aurora, CO

${ }^{\mathrm{h}}$ National Institute of Diabetes and Digestive and Kidney

Diseases, Bethesda, MD

'Department of Surgery, University of California,

Los Angeles, CA

'Department of Medicine, University of North Carolina, Chapel Hill, NC

${ }^{\mathrm{k}}$ Department of Medicine, University of Virginia, Charlottesville, VA

'Department of Internal Medicine, University of Michigan, Ann Arbor, MI

\section{* Corresponding author: Robert A. Fisher, rafisher@vcu.edu \\ †This is publication 19 of the Adult-to-Adult Living Donor Liver Transplantation Cohort Study.}

Hepatocellular carcinoma (HCC) represents an increasing fraction of liver transplant indications; the role of living donor liver transplant (LDLT) remains unclear. In the Adult-to-Adult Living Donor Liver Transplantation Cohort Study, patients with HCC and an LDLT or deceased donor liver transplant (DDLT) for which at least one potential living donor had been evaluated were compared for recurrence and posttransplant mortality rates. Mortality from date of evaluation of each recipient's first potential living donor was also analyzed. Unadjusted 5-year HCC recurrence was significantly higher after LDLT $(38 \%)$ than DDLT $(11 \%)$, ( $p=0.0004)$. After adjustment for tumor characteristics, HCC recurrence remained significantly different between LDLT and DDLT recipients (hazard ratio $(H R)=2.35 ; p=$ $0.04)$ for the overall cohort but not for recipients transplanted following the introduction of MELD prioritization. Five-year posttransplant survival was similar in LDLT and DDLT recipients from time of transplant (HR $=1.32 ; p=0.27$ ) and from date of LDLT evaluation (HR $=0.73 ; p=0.36$ ). We conclude that the higher recurrence observed after LDLT is likely due to differences in tumor characteristics, pretransplant HCC management and waiting time.

Key words: HCC, loco-regional therapy, MELD score, Milan criteria, recurrence, survival

Abbreviations: A2ALL, Adult to Adult Living Donor Transplantation Cohort Study; AFP, alphafetoprotein; $\mathrm{Cl}$, confidence interval; DDLT, deceased donor liver transplant; HCC, hepatocellular carcinoma; HR, hazard ratio; LDLT, living donor liver transplant; LRT, locoregional therapy; LT, liver transplant; MELD, model for end-stage liver disease; SD, standard deviation; TNM, tumor node metastasis; UCSF, University of California San Francisco.

Received 19 March 2012, revised 26 June 2012 and accepted for publication 27 June 2012

\section{Introduction}

Liver transplantation (LT) is a well-established therapeutic option in patients with unresectable hepatocellular carcinoma (HCC). The rate of recurrent $\mathrm{HCC}$ had been reported to be significantly higher following living donor liver transplant (LDLT) compared to deceased donor liver transplant (DDLT), even after adjustment for tumor stage (1-3). The reasons for this observation remain speculative. In the United States, enhanced prioritization for DDLT for candidates with HCC is limited to those fulfilling the Milan criteria, leaving LDLT as the only timely route to transplantation without the delay and uncertainty of downstaging for those with more advanced lesions. Anticipated prolonged waiting times for DDLT, with the risk of drop out due to tumor progression, may also provide motivation to pursue LDLT. Differences in expected waiting times in DDLT 


\section{Kulik et al.}

and LDLT can influence pretransplant management, specifically resulting in less application of loco-regional therapies (LRT) prior to LDLT due to shorter waiting times.

The Adult-to-Adult Living Donor Liver Transplantation Cohort Study (A2ALL) consortium previously reported a 3-year HCC recurrence probability of $29 \%$ following LDLT versus $0 \%$ following DDLT in a cohort of 92 recipients $(p=0.002)$ (1). The majority of these patients $(57 \%)$ were transplanted prior to the introduction of Model for End-Stage Liver Disease (MELD). Since the publication of our original report, the number of HCC patients who have undergone transplantation in the A2ALL study has more than doubled, and the median duration of follow-up following transplant has increased from 3.7 to 4.9 years. Moreover, the results reported in the earlier study were derived largely from a retrospective cohort. The main aims of this study were to compare recurrence and mortality following LDLT or DDLT. We also analyzed survival from the time of the donor's evaluation in an intention-to-treat analysis among those who had at least one potential living donor evaluated; we compared those who underwent LDLT versus those who did not receive an LDLT (LDLT candidates who received a DDLT plus those who had a potential living donor evaluated but were not transplanted at all).

\section{Methods}

The A2ALL Study Consortium consists of nine clinical centers and a data coordinating center. The overall design of the A2ALL Study has been described previously (4). Entry into the study was defined as the date of evaluation (history and physical examination) of the first potential live donor for a given transplant candidate. Subsequently, some patients underwent a LDLT and the remainder continued to wait for a DDLT. Data were obtained using retrospective chart review from January 1, 1998 to February 28, 2003, and using a combination of retrospective and prospective collection from February, 28, 2003 through August 31, 2010. The study was approved by the Institutional Review Board and Privacy Boards of the Data Coordinating Center at the University of Michigan and the nine participating centers. All patients in the prospective study provided written informed consent for participation.

Among a total of 1528 patients with a potential live donor evaluated between January 1, 1998 and August 31, 2009, 229 patients had known HCC prior to transplant, representing the cohort for this study. Since candidates with $\mathrm{HCC}$ within Milan criteria were given higher priority for DDLT in the MELD era beginning in February 2002, we also analyzed the results of subjects whose first potential donor underwent evaluation after the implementation of the MELD-based system.

Laboratory MELD score, alphafetoprotein (AFP) level, tumor characteristics and receipt of LRT were recorded at donor evaluation and at LT. The type of LRT and the number of treatments prior to LT were not standardized across centers. Computed tomography or MRI was performed every 3-6 months until LT. Tumor staging pre-LT according to the modified Tumor Node Metastasis (TNM) staging system, Milan criteria and University of California San Francisco (UCSF) criteria was based on local interpretation of radiologic imaging (5-7). Explant pathology was reviewed locally; the number, location and size of viable tumor nodules, presence of macroscopic/microscopic vascular invasion and Edmonson tumor grade were recorded. Patients were followed until the data censoring date-August 31, 2010 or death. Imaging and AFP levels were obtained posttransplant every 6 months for the first 2 years, and thereafter according to a schedule determined by the individual centers.

\section{Statistical analyses}

Descriptive statistics are presented as mean and standard deviation (SD) or percentage, and patient characteristics were compared by transplant type (LDLT or DDLT) using two-sample t-tests and chi-square tests. KaplanMeier estimates were computed for the three study outcomes, all computed as time from transplant: time to HCC recurrence, overall survival and recurrence-free survival. The outcomes for LDLT and DDLT patients were compared by a log-rank test. The probabilities of each of the competing risks of receiving LDLT or DDLT, dying on the waitlist and remaining alive without a transplant were estimated using the cumulative incidence function, computed by the SAS macro "comprisk" developed the Mayo Clinic (8). This function is displayed for both the pre-MELD and MELD eras.

Multivariable Cox models were used to assess predictors of HCC recurrence, overall survival and recurrence-free survival from time of transplant. Potential predictors included type of transplant, recipient age, recipient race, AFP at transplant, lab MELD at transplant, number of LRT prior to transplant, evaluation period (pre/post MELD), waiting time from donor evaluation to transplant, etiology of cirrhosis and the following explant characteristics: number of tumor nodules, size of largest nodule, tumor grade (G1-G3) and vascular invasion. Model selection was performed using the best subsets selection method (9). Hazard ratios (HR) and corresponding 95\% confidence intervals $(\mathrm{Cl})$ were reported for each prognostic factor in the final models. Subanalyses were also performed for patients evaluated in the MELD era (after February 28, 2002), and for patients after centers were considered to be experienced in LDLT (after 20 patients received LDLT).

Patient survival from the time of donor evaluation was analyzed to evaluate the benefit of LDLT compared to waiting for, and possibly receiving, a DDLT. This analysis used the sequential stratification modification of Cox regression, adjusted for baseline covariates of recipient age, recipient race, AFP, laboratory MELD, number of LRT and tumor stage, each assessed at the time of the subject's first donor evaluation (6).

The practice patterns of individual transplant centers, including criteria for transplantation (radiographic tumor staging and AFP levels at transplant) and the utilization of LRT prior to transplantation, as well as waiting time to transplant, differed among the centers. Statistical tests of center associations with posttransplant outcomes were limited by the small number of cases at individual transplant centers.

Data were analyzed by the Data Coordinating Center at the University of Michigan using SAS 9.2 software (SAS Institute Inc., Cary, NC, USA).

\section{Results}

\section{Patient characteristics at donor evaluation and at transplant}

Characteristics of the entire cohort at donor evaluation and at transplant are shown in Tables 1 and 2, respectively. There were no significant differences between LDLT and DDLT recipients in terms of demographic variables or calculated MELD score. At evaluation, the LDLT recipients had evidence of more advanced HCC with significantly higher AFP levels and a larger tumor burden 
Outcomes of LDLT and DDLT Recipients With HCC

Table 1: Characteristics of HCC patients at evaluation of the first living donor

\begin{tabular}{|c|c|c|c|c|}
\hline Characteristics $^{\dagger}$ & $\begin{array}{c}\text { LDLT }(n=100) \\
\text { Mean } \pm \text { SD or } \%\end{array}$ & $\begin{array}{c}\text { DDLT }(n=97) \\
\text { Mean } \pm \text { SD or } \%\end{array}$ & $\mathrm{p}$-Value $\ddagger$ & $\begin{array}{c}\text { No transplant }(n=32) \\
\text { Mean } \pm \text { SD or } \%\end{array}$ \\
\hline Male gender & 75 & 76 & 0.8332 & 69 \\
\hline Age (years) & $55.2 \pm 8.0$ & $53.9 \pm 8.5$ & 0.3070 & $54.7 \pm 10.5$ \\
\hline \multicolumn{5}{|l|}{ Race } \\
\hline White & 80 & 82 & 0.8879 & 75 \\
\hline African American & 2 & 2 & & 9 \\
\hline Other & 18 & 16 & & 16 \\
\hline HCV etiology of cirrhosis & 78 & 78 & 0.9981 & 72 \\
\hline \multicolumn{5}{|l|}{ AFP $(n g / m L)$} \\
\hline$<20$ & 48 & 62 & 0.0038 & 43 \\
\hline $20-400$ & 32 & 33 & & 37 \\
\hline$>400$ & 20 & 5 & & 20 \\
\hline Log AFP & $1.67 \pm 1.04$ & $1.18 \pm 0.70$ & 0.0002 & $1.81 \pm 1.17$ \\
\hline MELD & $12.1 \pm 4.5$ & $12.7 \pm 4.7$ & 0.3754 & $12.1 \pm 4.7$ \\
\hline Tumor stage & & & 0.0007 & \\
\hline TO* & 3 & 4 & & 4 \\
\hline T1 & 7 & 15 & & 7 \\
\hline $\mathrm{T} 2$ & 31 & 51 & & 56 \\
\hline T3 & 44 & 15 & & 26 \\
\hline $\mathrm{T} 4$ & 15 & 14 & & 7 \\
\hline Beyond Milan & 59 & 30 & $<0.0001$ & 33 \\
\hline Beyond UCSF & 42 & 20 & 0.0015 & 16 \\
\hline Diameter of largest nodule (cm) & $4.3 \pm 2.8$ & $3.2 \pm 1.7$ & 0.0009 & $3.9 \pm 2.9$ \\
\hline HCC ablation before donor evaluation & 45 & 65 & 0.0090 & 50 \\
\hline
\end{tabular}

${ }^{\dagger}$ Numbers of missing values for [LDLT, DDLT, no transplant] were: $[0,0,0]$ for gender, age and race, $[24,12,2]$ for HCV etiology of cirrhosis, and $[2-16,2-15,1-7]$ for all other variables.

$\ddagger$ p-values for comparison of LDLT and DDLT for age, log AFP, MELD and diameter of largest nodule computed from t-test; and for all other characteristics from the chi-square test.

* Of the seven TO patients, (3 LDLT, 3 DDLT, 1 no transplant), five patients received HCC treatment prior to enrollment and had been down staged to TO and the other two patients were diagnosed with HCC after enrollment.

(both a greater size of largest nodule and higher percentage exceeding Milan and UCSF criteria). LDLT recipients were significantly less likely to have received any form of ablative therapy (radiofrequency ablation, transarterial chemoembolization, radioembolization) and among those who received ablative therapy, LDLT recipients had less ablation sessions than DDLT recipients (1.2 vs. 1.8; $p=0.01)$. At transplant, differences in tumor staging based on radiologic imaging and LRT received persisted. However, the differences were attenuated when comparisons were based on transplant pathology (Table 2). LDLT patients had significantly shorter mean waiting times between donor evaluation and transplant compared to DDLT recipients (77.7 vs. 180.5 days, $p=$ $0.0004)$. Donor age was significantly younger and cold ischemia time significantly shorter among LDLT recipients. Similar differences were noted for patients in the MELD era ( $N=126)$ (Supporting Tables S1 and S2).

\section{Transplant timing and donor type}

Of the $229 \mathrm{HCC}$ patients, 100 received a LDLT, 97 a DDLT and 32 had not undergone transplantation at the time of data analysis. Among the 32 patients who did not receive a transplant, 27 died awaiting transplantation (median time from donor evaluation to death 11.9 months, range $0.3-79$ months) and 5 withdrew consent from the A2ALL (median time from donor evaluation to last follow up 8.6 months, range $0.2-50$ months). Of the 146 patients enrolled in the MELD era, 50 received a LDLT, 76 a DDLT and 20 had not undergone transplantation. The principal difference in the experience of transplant candidates between the two eras was a shift to DDLT in the MELD era (Figure 1). In the pre-MELD era, $60 \%$ of candidates received LDLT and $24 \%$ received DDLT, whereas in the MELD era, 35\% received LDLT and $54 \%$ received DDLT. The proportion who died without LT was $10 \%$ in both eras.

\section{Explant pathology}

LDLT recipients compared with DDLT recipients were more likely to have tumors exceeding the Milan $(56 \%$ vs. $44 \%, p=0.10)$ and UCSF criteria (48\% vs. $34 \%$, $p=0.06)$, to have significantly larger tumors $(p=0.01)$ and were more likely to have vascular invasion $(p=0.07)$ (Table 2). LDLT recipients in the MELD era also had significantly larger tumors ( $p=0.04$ ) and more frequent vascular invasion ( $p=0.10$ ) and sum of nodule sizes ( $p=$ 0.06) (Supporting Table S2). There was no significant difference between LDLT and DDLT groups regarding number of tumor nodules, histological grade or presence of bilobar disease. 
Table 2: Characteristics of HCC patients at transplant

\begin{tabular}{|c|c|c|c|}
\hline Characteristics $^{\dagger}$ & $\begin{array}{l}\operatorname{LDLT}(n=100) \\
\text { Mean } \pm \text { SD } \\
\quad \text { or } \%\end{array}$ & $\begin{array}{l}\text { DDLT }(n=97) \\
\text { Mean } \pm \text { SD } \\
\text { or } \%\end{array}$ & $p$-Value $\ddagger$ \\
\hline \multicolumn{4}{|l|}{ At transplant } \\
\hline AFP (ng/mL) & & & 0.0065 \\
\hline$<20$ & 48 & 59 & \\
\hline $20-400$ & 31 & 36 & \\
\hline$>400$ & 21 & 5 & \\
\hline Log AFP & $1.7 \pm 1.1$ & $1.2 \pm 0.7$ & 0.0003 \\
\hline Donor age (years) & $35.0 \pm 10.2$ & $42.3 \pm 17.7$ & 0.0019 \\
\hline $\begin{array}{l}\text { Cold ischemia } \\
\text { time (minutes) }\end{array}$ & $65.6 \pm 60.4$ & $409 \pm 195$ & $<0.0001$ \\
\hline MELD & $13.3 \pm 4.6$ & $13.8 \pm 6.4$ & 0.5067 \\
\hline Beyond Milan & 52 & 26 & 0.0003 \\
\hline Beyond UCSF & 35 & 14 & 0.0015 \\
\hline $\begin{array}{l}\text { HCC ablation } \\
\text { from donor } \\
\text { evaluation to LT }\end{array}$ & 31 & 49 & 0.0102 \\
\hline $\begin{array}{l}\text { HCC ablation ever } \\
\text { before LT }\end{array}$ & 59 & 73 & 0.0355 \\
\hline $\begin{array}{l}\text { No. of ablations } \\
\text { since diagnosis }\end{array}$ & $1.2 \pm 1.5$ & $1.8 \pm 1.7$ & 0.0137 \\
\hline $\begin{array}{l}\text { Days from donor } \\
\text { evaluation to } L T\end{array}$ & $77.7 \pm 106$ & $180.5 \pm 258$ & 0.0004 \\
\hline \multicolumn{4}{|l|}{ Explant pathology } \\
\hline Tumor stage & & & 0.2441 \\
\hline TO & 6 & 13 & \\
\hline $\mathrm{T} 1$ & 7 & 12 & \\
\hline T2 & 31 & 31 & \\
\hline T3 & 35 & 24 & \\
\hline T4 & 21 & 20 & \\
\hline Beyond Milan & 56 & 44 & 0.0963 \\
\hline Beyond UCSF & 48 & 34 & 0.0573 \\
\hline Bilobar HCC & 42 & 33 & 0.2160 \\
\hline $\begin{array}{l}\text { Diameter of } \\
\text { largest nodule } \\
\text { (cm) }\end{array}$ & $4.3 \pm 2.5$ & $3.5 \pm 1.9$ & 0.0140 \\
\hline $\begin{array}{l}\text { No. of tumor } \\
\text { nodules }\end{array}$ & $2.4 \pm 1.8$ & $2.1 \pm 1.7$ & 0.3189 \\
\hline Vascular invasion & 23 & 12 & 0.0658 \\
\hline $\begin{array}{l}\text { Total length of } \\
\text { nodules }(\mathrm{cm})\end{array}$ & $6.4 \pm 4.0$ & $5.4 \pm 3.3$ & 0.0906 \\
\hline Tumor grade & & & 0.9719 \\
\hline $\mathrm{G} 1$ & 29 & 29 & \\
\hline $\mathrm{G} 2$ & 54 & 53 & \\
\hline G3 & 16 & 18 & \\
\hline
\end{tabular}

${ }^{\dagger}$ Number of missing values for [LDLT, DDLT] were: [14,12] for AFP, $[1,24]$ for donor age, $[23,3]$ for cold ischemia time, [9,3] for Milan, $[16,5]$ for UCSF and $[0,0-1]$ for other transplant variables, and [2-7,4-14] for explants variables except for tumor grade, [32,35]. ${ }^{\ddagger} p$-values for comparison of LDLT and DDLT. Two-sample t-tests and chi-square tests were used for continuous variables and proportions, respectively.

HCC = hepatocellular carcinoma; LDLT = living donor liver transplant; $\mathrm{DDLT}=$ deceased donor liver transplant; $\mathrm{LT}=$ liver transplant.

\section{Post-LT HCC recurrence and survival}

Tumor recurrence by stage and era is shown in Table 3. In the pre-MELD era, HCC recurrence in DDLT recipients was limited to one patient who had T4b disease out of 21
(5\%), whereas 16 of 50 (32\%) LDLT recipients had HCC recurrence, including 5 (10\%) with less advanced HCC (TO, 1 or 2). In comparison, all recurrences in both LDLT and DDLT recipients in the MELD era occurred in those with advanced disease $(>\mathrm{T} 2)$. $\mathrm{HCC}$ recurrence was detected in 0 of $24(0 \%)$ LDLT recipients with early HCC $(\leq T 2), 11$ of $25(44 \%)$ LDLT with advanced HCC (>T2), 0 of $42(0 \%)$ DDLT recipients with early HCC, and 7 of 31 (23\%) DDLT with advanced HCC.

There were more HCC recurrences in LDLT compared to DDLT recipients for the entire cohort (28 LDLT, 8 DDLT recurrences, Figure $2 \mathrm{~A}$ ) as well as among the subset of MELD era patients (Figure 2B). After a median posttransplant follow-up of 4.3 years in DDLT and 5.9 years in LDLT recipients, the 5-year unadjusted probability of HCC recurrence was $38 \%$ in LDLT recipients and $11 \%$ in DDLT recipients $(p=0.0004)$. After adjusting for explant tumor size, number of tumor nodules and presence of vascular invasion, HCC recurrence rates remained higher in LDLT recipients $(H R=2.35,95 \% \mathrm{Cl} 1.04-5.35 ; \mathrm{p}=0.04)$ (Figure 2C, Table 4, panel A). However, in the MELD era, the association of LDLT with HCC recurrence was present but not statistically significant in a similar model adjusted for tumor characteristics $(\mathrm{HR}=1.36,95 \% \mathrm{Cl}$ 0.47-3.93; $\mathrm{p}$ $=0.57$ ) (Table 4, panel B).

The difference in $\mathrm{HCC}$ recurrence rates between LDLT and DDLT recipients in the entire cohort was most striking during the first year posttransplant $(15 \%$ vs. $0 \%$; $p<$ 0.0001). Conditional on being recurrence-free at 1 year, and after adjusting for explant tumor size, number of tumor nodules and presence of vascular invasion, the subsequent recurrence risks for LDLT and DDLT were almost identical $(\mathrm{HR}=1.20,95 \% \mathrm{Cl} 0.48-2.98 ; \mathrm{p}=0.69)$. LDLT recipients with early $\mathrm{HCC}$ recurrence were significantly more likely to have a larger tumor diameter $(p=0.0008)$, a higher AFP level at the time of transplant $(p=0.0002)$, and presence of grade $3 \mathrm{HCC}$ on explant liver $(p=0.0004)$ (Supporting Figure S1).

Survival was similar between LDLT and DDLT recipients for the entire cohort as well as the MELD era patients (Figures $3 \mathrm{~A}$ and $3 \mathrm{~B}$ ). The 5-year unadjusted survival probability was $59 \%$ in LDLT recipients and $66 \%$ in DDLT recipients $(H R=1.32 ; p=0.27)$. The type of transplant did not predict post-LT mortality in multivariable Cox models for the entire cohort $(\mathrm{HR}=1.25,95 \% \mathrm{Cl} 0.69-2.26$; $\mathrm{p}=0.46)$ or in the subset of patients in the MELD era $(H R=0.85,95 \% \mathrm{Cl} 0.33-2.19 ; p=0.74)$. Similarly, there was no difference in recurrence-free survival between LDLT and DDLT recipients for the entire cohort $(H R=$ $1.51,95 \% \mathrm{Cl} 0.88-2.59 ; \mathrm{p}=0.13$ ) or for those in the MELD era $(H R=1.23,95 \% \mathrm{Cl} 0.55-2.74 ; p=0.61)$ (Figures $4 \mathrm{~A}$ and $4 \mathrm{~B}$ ).

A total of 39 LDLT and 27 DDLT patients died by the end of the study. HCC recurrence was the cause of death in 
A

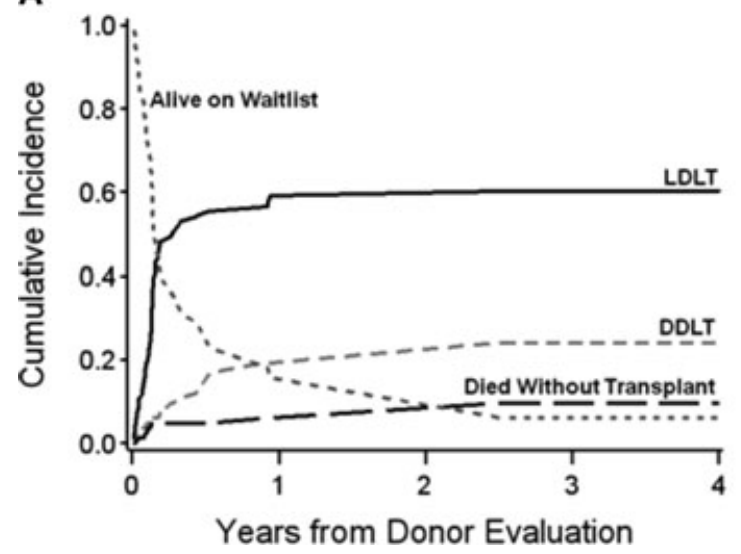

B

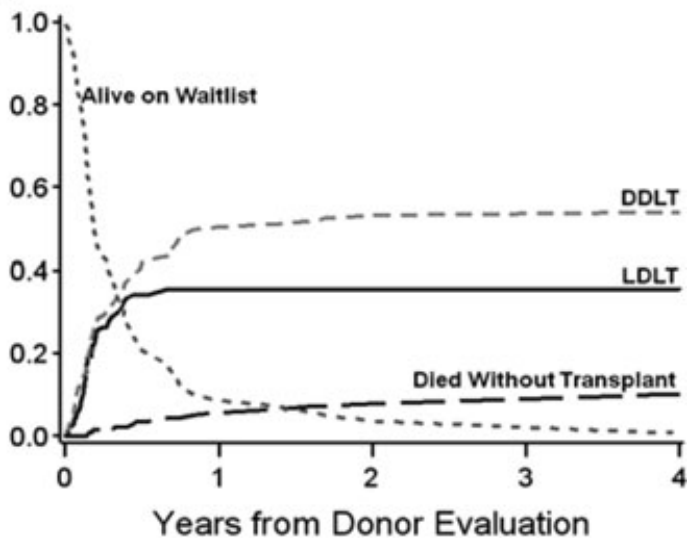

Figure 1: Cumulative probability over time of LDLT, DDLT, remaining alive on the waitlist and death without transplant, from the first living donor evaluation (based on the cumulative incidence function) for (A) HCC patients in the pre-MELD Era and (B) HCC patients in the MELD era.

24 patients (18 LDLT, 6 DDLT). Unadjusted survival from the time of HCC recurrence to death was not statistically significantly different in the LDLT and DDLT patients (median time to death 23 months LDLT, 36 months DDLT; $p=0.14)$. The causes of death in the remaining patients included: cardiac arrest (2 LDLT, 3 DDLT), recurrent hepatitis (2 LDLT, 2 DDLT), infection (4 LDLT, 2 DDLT), lymphoma and other malignancies (0 LDLT, 3 DDLT), respiratory failure (3 LDLT, 1 DDLT), other (5 LDLT, 3 DDLT) and unknown (5 LDLT, 7 DDLT).

\section{Survival from time of potential living donor evaluation} To assess whether receiving an LDLT was associated with a survival benefit over waiting for a DDLT, analysis from the time donor evaluation was performed. For this analysis, 219 patients with HCC known at the time of donor evaluation were included and 10 patients with HCC diagnosed after donor evaluation were excluded. The adjusted risk of death from the time of donor evaluation was similar for patients who received LDLT versus nonLDLT (patients who received DDLT and those who continued to wait for but did not receive any $L T$ ) for the entire cohort $(\mathrm{HR}=0.73,95 \% \mathrm{Cl} 0.36-1.45 ; \mathrm{p}=0.36)$ and in the MELD era $(H R=0.90,95 \% \mathrm{Cl} 0.35-2.33 ; \mathrm{p}=0.83)$ (Figures 5A and 5B).

\section{Factors associated with HCC recurrence, survival and recurrence free survival posttransplant}

In addition to transplant type, independent predictors of HCC recurrence for the entire cohort included size of largest tumor nodule and number of tumor nodules on explant liver (Table 4, panel A). These tumor characteristics as well as the presence of vascular invasion predicted $\mathrm{HCC}$ recurrence in the MELD era (Table 4, panel B).

Post-LT mortality for the entire cohort was predicted by AFP level at transplant $(\mathrm{HR}=1.35,95 \% \mathrm{Cl} 1.07-1.71 ; \mathrm{p}=$ 0.01) (Table 4, panel A) and for the MELD era by the diameter of the largest nodule on explant and vascular invasion (Table 4, panel B).

Similarly, AFP at transplant, greater size of the largest tumor nodule, and number of tumor nodules on explant predicted recurrence free survival for the entire cohort, while greater size of the largest tumor nodule and presence of vascular invasion were predictors of

Table 3: HCC recurrence post-LT by type of transplant, tumor stage on explant and MELD era

\begin{tabular}{|c|c|c|c|c|c|c|c|}
\hline \multirow[b]{2}{*}{ Tumor stage on explant } & \multicolumn{3}{|c|}{ LDLT } & \multicolumn{3}{|c|}{ DDLT } & \multirow[b]{2}{*}{ Overall } \\
\hline & Pre-MELD & MELD & All & Pre-MELD & MELD & All & \\
\hline T0 & $1 / 3$ & $0 / 3$ & $1 / 6$ & $0 / 2$ & $0 / 10$ & $0 / 12$ & $1 / 18$ \\
\hline T1 & $0 / 3$ & $0 / 4$ & $0 / 7$ & $0 / 2$ & $0 / 9$ & $0 / 11$ & $0 / 18$ \\
\hline T2 & $4 / 13$ & $0 / 17$ & $4 / 30$ & $0 / 6$ & $0 / 23$ & $0 / 29$ & $4 / 59$ \\
\hline T3 & $6 / 17$ & $6 / 17$ & $12 / 34$ & $0 / 5$ & $5 / 17$ & $5 / 22$ & $17 / 56$ \\
\hline T4a & $3 / 10$ & $3 / 6$ & $6 / 16$ & $0 / 4$ & $1 / 13$ & $1 / 17$ & $7 / 33$ \\
\hline $\mathrm{T} 4 \mathrm{~b}$ & $2 / 3$ & $2 / 2$ & $4 / 5$ & $1 / 1$ & $1 / 1$ & $2 / 2$ & $6 / 7$ \\
\hline Missing & $0 / 1$ & $1 / 1$ & $1 / 2$ & $0 / 1$ & $0 / 3$ & $0 / 4$ & $1 / 6$ \\
\hline Total & $16 / 50(32 \%)$ & $12 / 50(24 \%)$ & $28 / 100(28 \%)$ & $1 / 21(5 \%)$ & $7 / 76(9 \%)$ & $8 / 97(8 \%)$ & $36 / 197$ (18\%) \\
\hline
\end{tabular}


A

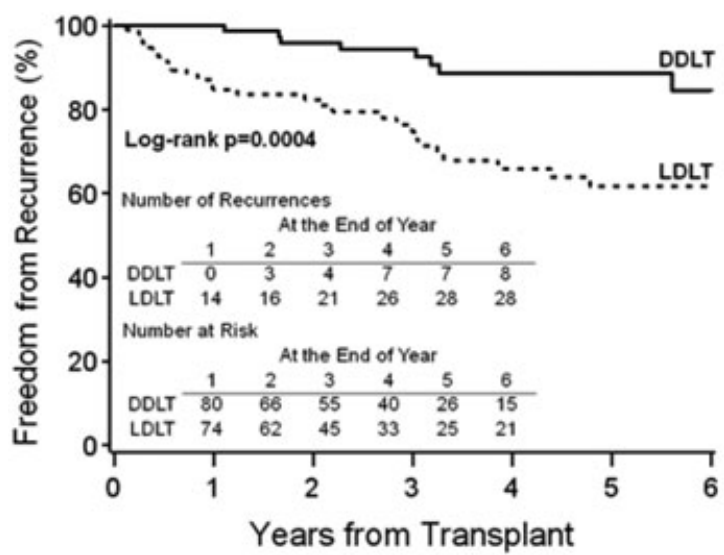

C

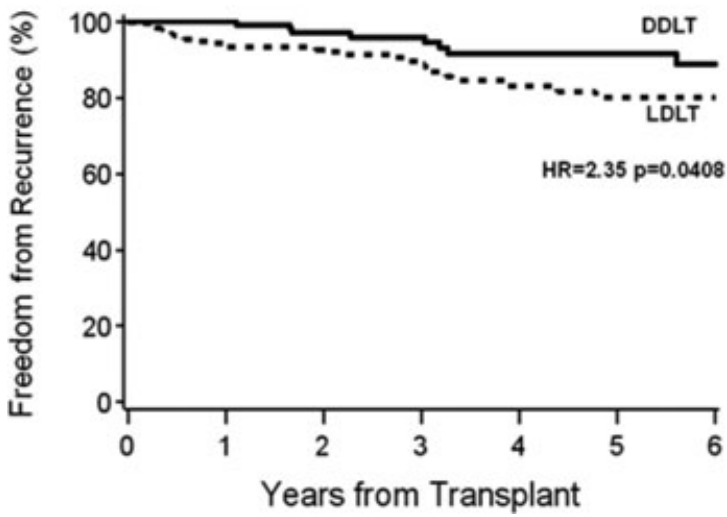

B

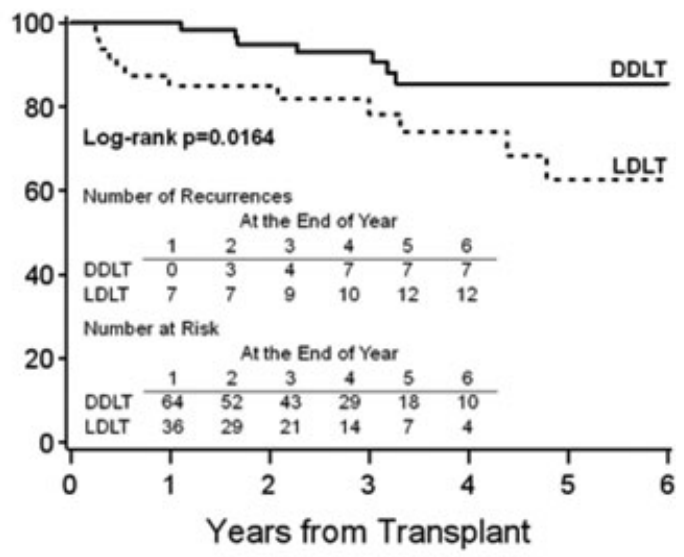

Figure 2: Probability of freedom from HCC recurrence by time since LDLT or DDLT for (A) all HCC patients (unadjusted), (B) HCC patients in the MELD era (unadjusted) and (C) all HCC patients (adjusted based on a Cox regression model, with both LDLT and DDLT groups presented for the overall average baseline characteristic values of two liver nodules, largest nodule $3.5 \mathrm{~cm}$ and without vascular invasion).

Table 4: Cox regression models predicting HCC recurrence, death and the combined outcome posttransplant

Panel A: for all HCC patients

\begin{tabular}{|c|c|c|c|c|c|c|c|c|c|}
\hline \multirow[b]{2}{*}{ Predictors } & \multicolumn{3}{|c|}{ HCC recurrence only } & \multicolumn{3}{|c|}{ Death } & \multicolumn{3}{|c|}{ HCC recurrence or death } \\
\hline & $\mathrm{HR}$ & $95 \% \mathrm{Cl}$ & p-Value & $\mathrm{HR}$ & $95 \% \mathrm{Cl}$ & p-Value & $\mathrm{HR}$ & $95 \% \mathrm{Cl}$ & p-Value \\
\hline LDLT versus DDLT & 2.35 & $(1.04,5.35)$ & 0.0408 & 1.25 & $(0.69,2.26)$ & 0.4572 & 1.51 & $(0.88,2.59)$ & 0.1326 \\
\hline Number of $\mathrm{HCC}$ nodules in the liver & 1.42 & $(1.17,1.71)$ & 0.0003 & - & - & - & - & - & - \\
\hline Diameter of largest nodule (cm) & 1.27 & $(1.13,1.42)$ & $<0.0001$ & 1.11 & $(1.00,1.23)$ & 0.0610 & 1.14 & $(1.04,1.26)$ & 0.0076 \\
\hline Vascular invasion & 1.96 & $(0.94,4.11)$ & 0.0736 & 1.68 & $(0.92,3.07)$ & 0.0918 & 1.97 & $(1.13,3.44)$ & 0.0170 \\
\hline AFP at transplant $(\mathrm{ng} / \mathrm{mL}, \mathrm{log})$ & - & - & - & 1.35 & $(1.07,1.71)$ & 0.0126 & 1.29 & $(1.02,1.63)$ & 0.0348 \\
\hline
\end{tabular}

Panel B: for MELD era patients

\begin{tabular}{|c|c|c|c|c|c|c|c|c|c|}
\hline \multirow[b]{2}{*}{ Predictors } & \multicolumn{3}{|c|}{ HCC recurrence only } & \multicolumn{3}{|c|}{ Death } & \multicolumn{3}{|c|}{$\mathrm{HCC}$ recurrence or death } \\
\hline & $\mathrm{HR}$ & $95 \% \mathrm{Cl}$ & $\mathrm{p}$-Value & $\mathrm{HR}$ & $95 \% \mathrm{Cl}$ & $\mathrm{p}$-Value & $\mathrm{HR}$ & $95 \% \mathrm{Cl}$ & p-Value \\
\hline LDLT versus DDLT & 1.36 & $(0.47,3.93)$ & 0.5687 & 0.85 & $(0.33,2.19)$ & 0.7410 & 1.23 & $(0.55,2.74)$ & 0.6094 \\
\hline Number of $\mathrm{HCC}$ nodules in the liver & 1.70 & $(1.30,2.22)$ & 0.0001 & - & - & - & - & - & - \\
\hline Diameter of largest nodule (cm) & 1.28 & $(1.07,1.53)$ & 0.0066 & 1.19 & $(1.03,1.38)$ & 0.0199 & 1.21 & $(1.06,1.37)$ & 0.0036 \\
\hline Vascular invasion & 3.04 & $(1.10,8.39)$ & 0.0314 & 3.45 & $(1.43,8.34)$ & 0.0059 & 4.60 & $(2.07,10.2)$ & 0.0002 \\
\hline AFP at transplant $(\mathrm{ng} / \mathrm{mL}, \mathrm{log})$ & - & - & - & 1.34 & $(0.92,1.94)$ & 0.1274 & 1.14 & $(0.80,1.64)$ & 0.4631 \\
\hline
\end{tabular}


A

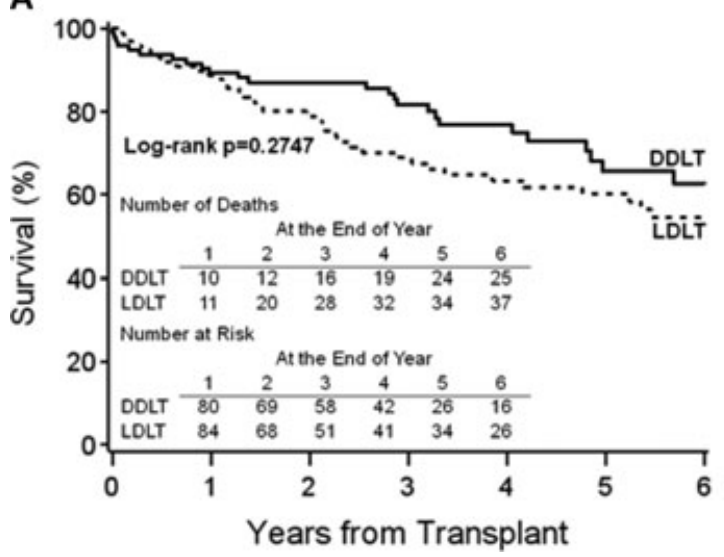

B

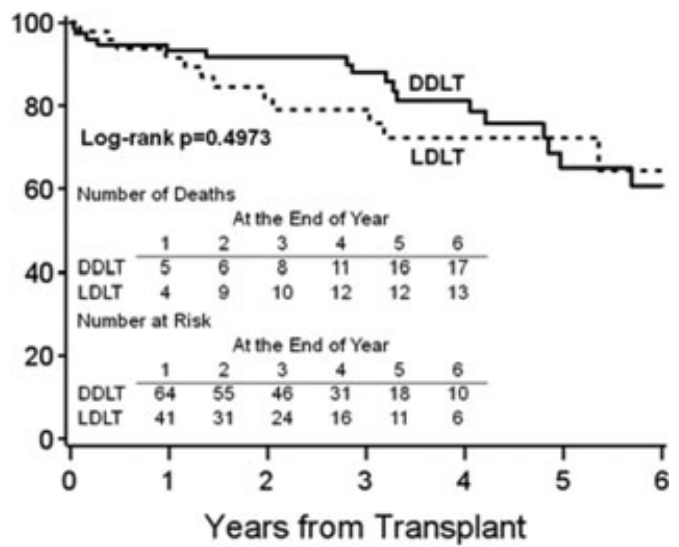

Figure 3: Unadjusted probability of patient survival by time since LDLT or DDLT for (A) all HCC patients and (B) HCC patients in the MELD era.

A

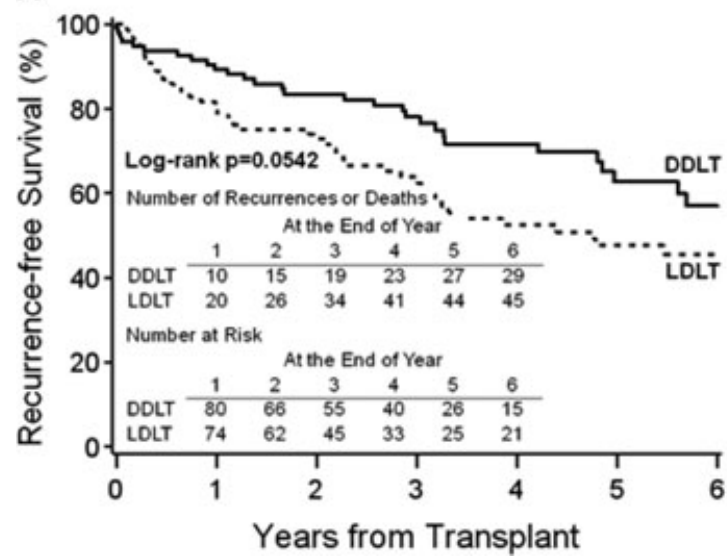

B

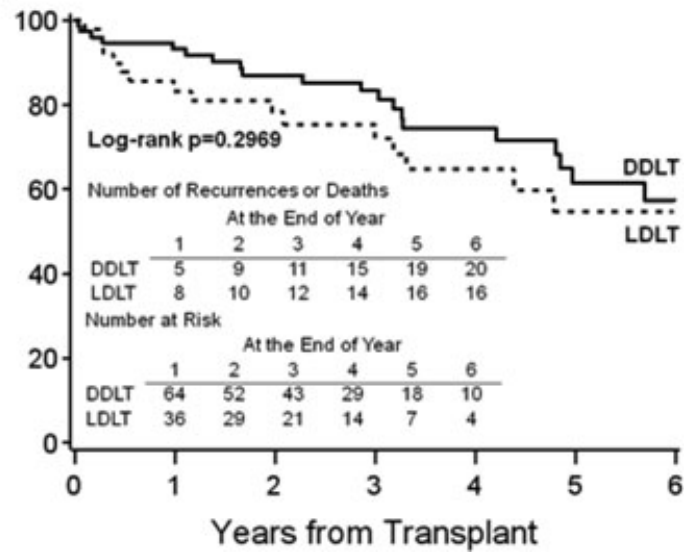

Figure 4: Unadjusted probability of recurrence-free survival by time since LDLT or DDLT for (A) all HCC patients and (B) HCC patients in the MELD era.

A

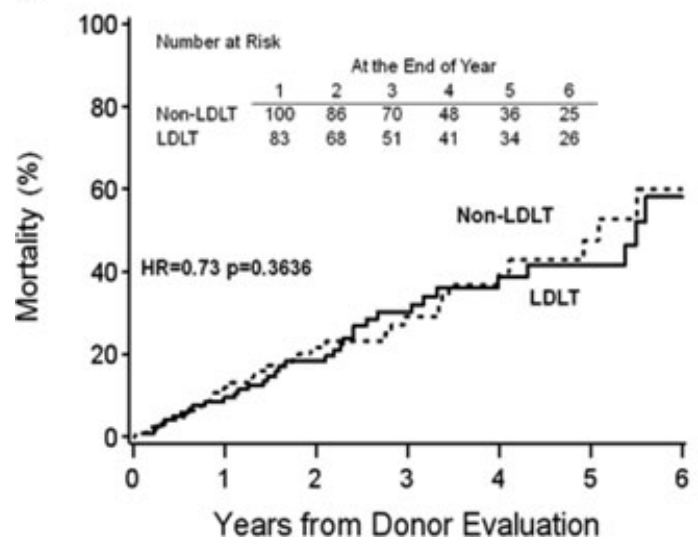

B

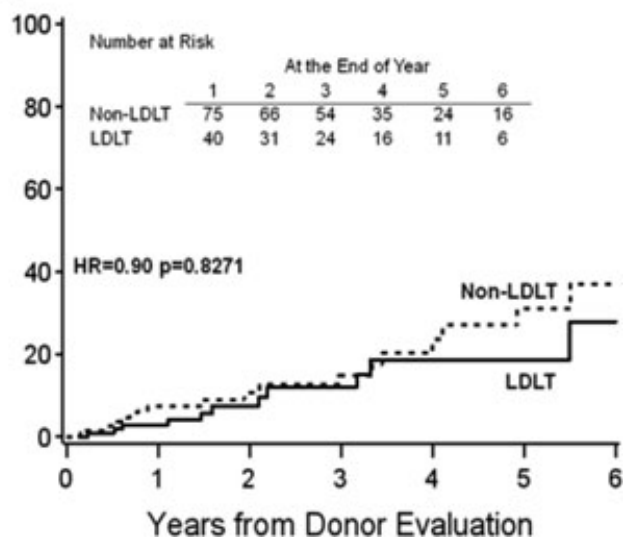

Figure 5: Adjusted probability of patient mortality by time from first living donor evaluation, for (A) all HCC patients and (B) HCC patients in the MELD era, both based on Cox regression models with both LDLT and DDLT groups presented for the average baseline characteristic values (55 years old, beyond Milan, AFP $=10$, lab MELD $=12$ and without ablation). 


\section{Kulik et al.}

recurrence free survival for the MELD era patients (Table 4, panels $A$ and $B$ ).

\section{Discussion}

Analysis of the early A2ALL experience which accrued prior to implementation of MELD found a significantly higher rate of HCC recurrence among LDLT recipients compared to DDLT recipients. Implementation of MELD had a major impact on prioritization for patients with HCC within Milan criteria, resulting in a higher percentage of patients undergoing DDLT and a lower percentage undergoing LDLT compared to the pre-MELD era (Figure 1). The current analysis included a substantially larger sample size with $64 \%$ enrolled in the MELD era. The unadjusted excess risk of HCC recurrence after LDLT compared to DDLT was attenuated, but remained significant in the MELD era. Independent predictors of $\mathrm{HCC}$ recurrence for the entire cohort included type of transplant, number of tumor nodules and diameter of the largest nodule on explant liver.

In the A2ALL retrospective study, LDLT was associated with increased HCC recurrence after stratifying by stage at explant; a multivariable analysis to determine factors associated with HCC recurrence in this cohort was not feasible due to no recurrence in the DDLT group. The lack of HCC recurrence among DDLT recipients and higher than expected recurrence rate among LDLT recipients with early HCC (6/23) led to the proposal of 'fast-tracking' as an explanation for these findings (10). Fast-tracking a patient to LDLT could theoretically negatively impact outcomes by inclusion of patients with inherently more aggressive tumor biology. In the current study, the failure to demonstrate a significant difference in $\mathrm{HCC}$ recurrence between LDLT and DDLT in the MELD era, after controlling for tumor characteristics, may be a reflection of extreme MELD era prioritization for DDLT mimicking the effect of LDLT 'fast-tracking' and producing more similar recurrence rates.

In our current cohort, LDLT was significantly associated with HCC recurrence even after adjusting for tumor characteristics for the entire cohort. The role of tumor biology on HCC recurrence was most pronounced among LDLT recipients with recurrence within 1-year posttransplant. Fourteen (15\%) LDLT, but none of the DDLT recipients, had HCC recurrence within the first year posttransplant. Tumor characteristics consistent with an aggressive phenotype were noted in the patients with early recurrence; larger tumors, higher AFP and grade $3 \mathrm{HCC}$. It has been postulated that the rapidity of liver regeneration can have a stimulatory effect on residual tumor cells, leading to higher HCC recurrence associated with partial transplants. A recent animal model supports this hypothesis (11).

An extensive experience of LDLT has been reported from Asia where live donors are the predominant source of grafts for transplant. Several authors have demonstrated tumor size $>5 \mathrm{~cm}$ in LDLT to be an independent predictor for HCC recurrence (12-18). This is in line with our experience that demonstrates tumor size to be an independent predictor of $\mathrm{HCC}$ recurrence. The significantly higher 5-year $\mathrm{HCC}$ recurrence rate in LDLT compared to DDLT recipients is related to greater tumor burden in LDLT recipients ( $p=$ 0.03).

Recently, Bhangui et al. reported no difference in recurrence rates in LDLT versus DDLT recipients, but did note a trend for poorer outcomes in LDLT recipients whose tumors exceeded the Milan criteria (19). A major difference in their cohort compared to ours was that the tumor characteristics and pretransplant treatments were not significantly different between DDLT and LDLT groups. This is likely related to DDLT not being limited to patients within the Milan criteria in this European cohort. In contrast, DDLT prioritization in the United States is limited to those fulfilling Milan criteria.

Despite having more advanced tumors, the LDLT recipients were less likely to receive pretransplant LRT and had fewer treatment sessions when rendered. A lack of response to LRT has been shown to be a predictor of HCC recurrence in DDLT (19-21). LRT followed by a time period to assess tumor response ('ablate and wait' approach) may allow the tumor an opportunity to declare a potentially aggressive behavior prior to LT $(22,23)$. However, a standardized approach of LRT has not been widely adopted among transplant centers. The receipt of LRT did not emerge as a predictor of outcome in our cohort, possibly due to heterogeneity in use of LRT among centers and the fact that we controlled for the effect of LRT by analyzing explant tumor burden. LDLT recipients in our study also had significantly higher AFP levels at donor evaluation and at transplant compared to the DDLT group. Decline in AFP levels in response to LRT has been shown to predict improved overall and post-LT survival rates (10,24-27). Merani et al. reported that an AFP $\leq 400 \mathrm{ng} / \mathrm{mL}$ closest to LT predicted better survival including patients who had an initial AFP $>1000 \mathrm{ng} / \mathrm{mL}$ and a decline to $\leq 400 \mathrm{ng} / \mathrm{mL}$ with LRT (28). Differences in HCC recurrence based on changes in AFP levels could not be analyzed in our study because serial AFP levels were not collected.

The only independent predictor of survival was AFP at the time of transplant in the entire cohort and tumor size and vascular invasion in the MELD era. Importantly, posttransplant survival was comparable between DDLT and LDLT recipients even though LDLT recipients in the entire cohort had a significantly higher rate of $\mathrm{HCC}$ recurrence after adjusting for tumor characteristics. Our data suggest that LDLT may confer a survival advantage that is unrelated to HCC recurrence.

Vakili et al. reported a significantly improved 5-year survival in LDLT compared to DDLT recipients (81\% vs. 58\%; 
$\mathrm{p}=0.023)$ despite a significantly higher HCC recurrence rate among the LDLT group (29\% vs. $12 \%$; $p<0.05)$ (3). Improved survival in the LDLT group may be related to the benefits of a superior quality of the graft due to potentially younger donors and less cold ischemia time. In contrast to the A2ALL cohort, tumor characteristics including AFP levels were not significantly different between the two groups. The only significant predictor of survival in their study was the presence of poorly differentiated HCC leading to death from HCC recurrence within 2.8 years of LDLT. To this end, some centers have adopted tumor grade as a selection criterion for transplant regardless of tumor size and number (29-31). Tumor grade was not used as a selection criterion for transplant in our cohort and was not significantly different between LDLT and DDLT recipients, except among the LDLT recipients with early HCC recurrence. Although tumor grade was missing in 34\% of patients, when tested in subset models in the entire cohort it was a significant predictor of HCC recurrence ( $p=0.0003$ ), and had a nonstatistically significant trend for survival $(p=0.08)$.

An important consideration in the decision of LDLT and DDLT is survival from the time of donor evaluation, taking into account drop out and wait list mortality. In this study, there was no difference in survival between patients who did or did not receive LDLT. However, in another analysis of the A2ALL data, a survival benefit of LDLT was observed in patients with HCC and a laboratory MELD exceeding 15 (32). It is plausible that higher MELD scores prohibited LRT leading to an increased risk of drop out in those without a live donor. Alternatively, patients with higher MELD may succumb to liver failure if timely transplant is not feasible. Data from the A2ALL suggest that HCC patients who are most likely to benefit from LDLT are those with T3 or T4a tumors who are not eligible for MELD upgrade and those with an unadjusted MELD > 15.

The strengths of this study include a large number of patients ( $N=229$ ), a long duration of follow-up (median 4.95 years), and most importantly, LDLT and DDLT recipients were comparable in that they all had a potential donor. The limitations include variability in center practices regarding selection of candidates for LDLT based on tumor stage, differences in use of LRT and inevitable differences in waiting time for DDLT in each region.

In conclusion, we believe that the higher $\mathrm{HCC}$ recurrence rate in LDLT recipients is due primarily to more advanced HCC and less liver directed HCC therapy compared to DDLT, as opposed to the type of transplant. Despite higher recurrence rates, overall survival was not significantly different from DDLT. With the risk posed to the donor and the benefit of an expedited transplant via LDLT being negated with the institution of HCC MELD upgrade for patients with T2 tumors, it begs the question of the current role for LDLT in HCC. Our data suggest that patients who are most likely to derive a benefit from LDLT are those with tumors exceeding the Milan criteria. However, our findings also support the need for a selection process in order to minimize HCC recurrence posttransplant. A mandated period of observation after pre-transplant LRT to document response based on radiology and a decline in AFP should be considered a prerequisite before proceeding with LDLT. Others, as well as our data, support an elevated AFP level at the time of transplant predicting overall survival $(28,33)$. Changes in United Network for Organ Sharing prioritization for HCC, such as a mandated waiting period before receiving a HCC MELD upgrade along with an abolition of prioritization if AFP level exceeds $500 \mathrm{ng} / \mathrm{mL}$ in an attempt to eliminate aggressive phenotypes, might motivate increased use of LDLT for HCC. Therefore, the transplant community will need to even more so be judicious in the use of LDLT for HCC. In the absence of a selection process for LDLT, both donors and recipients should be informed of the increased risk of HCC recurrence post-transplant when this procedure is performed in patients with tumors beyond the Milan criteria.

\section{Acknowledgments}

The patients participating in this trial were enrolled in the $\mathrm{NIH}$-sponsored Adult-to-Adult Living Donor Liver Transplantation Cohort Study. This study was supported by the National Institute of Diabetes and Digestive and Kidney Diseases through cooperative agreements (NIDDK grant numbers: U01-DK62536, U01-DK62444, U01-DK62467, U01-DK62483, U01DK62484, U01-DK62494, U01-DK62496, U01-DK62498, U01-DK62505, U01-DK62531)

This study was presented in part at the 61st annual meeting of the American Association for the Study of Liver Diseases, Boston, MA. November 2010. This study was supported by National Institute of Diabetes \& Digestive \& Kidney Diseases through cooperative agreements (listed below). Additional support was provided by Health Resources and Services Administration (HRSA), and the American Society of Transplant Surgeons (ASTS).

The following individuals were instrumental in the planning, conduct and/or care of patients enrolled in this study at each of the participating institutions as follows:

Columbia University Health Sciences, New York, NY (DK62483): principa investigator, Jean C. Emond, M.D.; coprincipal investigator, Robert S. Brown Jr, M.D., M.P.H.; study coordinators, Scott Heese, B.A., and Jonah S. Zaretsky, B.A.

Northwestern University, Chicago, IL (DK62467): principal investigator Michael M. I. Abecassis, M.D., M.B.A.; coprincipal investigator, Laura M. Kulik, M.D.; study coordinator, Patrice Al-Saden, R.N., C.C.R.C.

University of Pennsylvania Health System, Philadelphia, PA (DK62494): principal investigator, Abraham Shaked, M.D., Ph.D.; coprincipal investigator, Kim M. Olthoff, M.D.; study coordinators, Brian Conboy, P.A., M.B.A., and Mary Shaw, R.N., B.B.A.

University of Colorado Health Sciences Center, Denver, CO (DK62536): principal investigator, Gregory T. Everson, M.D.; coprincipal investigator, Igal Kam, M.D.; study coordinators, Carlos Garcia, B.S., Anastasia Krajec, R.N., and Andrea Herman, R.N. 


\section{Kulik et al.}

University of California Los Angeles, Los Angeles, CA (DK62496): principal investigator, Johnny C. Hong, M.D.; coprincipal investigator, Ronald W. Busuttil, M.D., Ph.D.; study coordinator, Janet Mooney, R.N., B.S.N.

University of California San Francisco, San Francisco, CA (DK62444): principal investigator, Chris E. Freise, M.D., F.A.C.S.; coprincipal investigator, Norah A. Terrault, M.D.; study coordinator, Dulce MacLeod, R.N.

University of Michigan Medical Center, Ann Arbor, MI (DK62498): principal investigator, Robert M. Merion, M.D.; data coordinating center staff, Anna S. F. Lok, M.D., Akinlolu O. Ojo, M.D., Ph.D., Brenda W. Gillespie, Ph.D., Margaret Hill-Callahan, B.S., L.S.W., Charlotte J. Arrington, M.P.H, Beth Golden, B.S.N, C.C.R.C, Tania Chowdhury, M.S., Terese Howell, B.S., C.C.R.C., Lisa Holloway, B.S., C.C.R.C., Monique Lowe, M.S., Abby Smith, M.S., and Yevgeniya Abramovich, B.A.

University of North Carolina, Chapel Hill, NC (DK62505): principal investigator, Paul H. Hayashi, M.D., M.P.H.; study coordinator, Tracy Russell, M.A.

University of Virginia (DK62484): principal investigator, Carl L. Berg, M.D., Duke University; study coordinator, Jaye Davis, R.N.

Medical College of Virginia Hospitals, Virginia Commonwealth University, Richmond, VA (DK62531): principal investigator, Robert A. Fisher, M.D., F.A.C.S.; coprincipal investigator, R. Todd Stravitz, M.D.; study coordinators, April Ashworth, R.N., and Andrea Lassiter, B.S.

Division of Digestive Diseases and Nutrition, National Institute of Diabetes and Digestive and Kidney Diseases, Bethesda, MD: James E. Everhart, M.D., M.P.H., Averell Sherker, M.D., and Jay H. Hoofnagle, M.D.

\section{Disclosure}

The authors of this manuscript have conflicts of interest to disclose as described by the American Journal of Transplantation.

Laura M. Kulik-Advisory Committees: Bayer/Onyx; Grant/Research Support: Bayer/Onyx; Speaking and Teaching: Bayer/Onyx.

Anna S. Lok-Consulting: Roche, Gilead, Bristol-Myers Squibb, Bayer; Grant/Research Support: Bristol-Myers Squibb, GlaxoSmithKline, Schering-Plough, Roche, Gilead.

Robert S. Brown-Grant/Research Support: Gilead, GSK, Novartis, Salix, Schering/Merck, Vertex; Speaking and Teaching: Salix Pharmaceuticals, Roche/Genentech, Gilead, Schering/Merck.

Greg T. Everson-Advisory Committee or Review Panels: Roche, Merck, Three Rivers, Hep C Connection; Board membership: HepQuant LLC, PSC partners; Consulting: Genentech; Grant/Research Support: Roche, Pharmassett, Vertex, GSK, Schering-Plough, Bristol-Myers Squibb, Tibotec, Human Genome Sciences/Novartis, Globelmmune, OrthoBiotech, Medtronic, Pfizer, Eisai, Abbott, Conatus, ZymoGenetics, PSC Partners; Management Position: HepQuant LLC; Patent Held/Filed: University of Colorado.
These authors have nothing to disclose: Robert A. Fisher, Del R. Rodrigo, Christopher E. Freise, Abraham Shaked, Johnny C. Hong, Paul H. Hayashi, Carl L. Berg, James E. Everhart.

\section{References}

1. Fisher RA, Kulik LM, Freise $C E$, et al. Hepatocellular carcinoma recurrence and death following living and deceased donor liver transplantation. Am J Transplant 2007; 7: 1601-1608.

2. Lo CM, Fan ST, Liu CL, Chan SC, Ng IO, Wong J. Living donor versus deceased donor liver transplantation for early irresectable hepatocellular carcinoma. Br J Surg 2007; 94: 78-86.

3. Vakili K, Pomposelli JJ, Cheah YL, et al. Living donor liver transplantation for hepatocellular carcinoma: Increased recurrence but improved survival. Liver Transpl 2009; 15: 1861-1866.

4. Olthoff KM, Merion RM, Ghobrial RM, et al. Outcomes of 385 adult-to-adult living donor liver transplant recipients: A report from the A2ALL Consortium. Ann Surg 2005; 242: 314-323.

5. American Liver Tumor Study Group: A randomized prospective multi institutional trial of orthotopic liver transplantation or partial hepatic resection with or without adjuvant chemotherapy for hepatocellular carcinoma. Investigator Booklet and Protocol, 1998. United Network for Organ Sharing. Policy 3.6.4.4, 1998.

6. Mazzaferro V, Regalia E, Doci R, et al. Liver transplantation for the treatment of small hepatocellular carcinomas in patients with cirrhosis. N Engl J Med 1996; 334: 693-699.

7. Yao FY, Ferrell L, Bass NM, et al. Liver transplantation for hepatocellular carcinoma: Expansion of the tumor size limits does not adversely impact survival. Hepatol 2001; 33: 13941403.

8. Carter RE, Lin Y, Lipsitz SR, Newcombe RG, Hermayer KL. Relative risk estimated from the ratio of two median unbiased estimates. J R Stat Soc Ser C Appl Stat 2010; 59: 657-671.

9. Myers, RH. Classical and Modern Regression (Bookware Companion Series). Duxbury Advanced Series in Statistics and Decision Sciences. Boston: PWS Pub. Co.; 2nd edition (August 1997)

10. Kulik L, Abecassis M. Living donor liver transplantation for hepatocellular carcinoma. Gastroenterology 2004; $127(5$ Suppl 1): S277S282.

11. Shi JH, Huitfeldt HS, Suo ZH, Line PD. Growth of hepatocellular carcinoma in the regenerating liver. Liver Transpl 2011; 17: 866874.

12. Schaubel DE, Wolfe RA, Port FK. A sequential stratification method for estimating the effect of a time-dependent experimental treatment in observational studies. Biometrics 2006; 62: 910917.

13. Todo S, Furukawa H, Tada M. Japanese Liver Transplantation Study Group. Extending indication: Role of living donor liver transplantation for hepatocellular carcinoma. Liver Transpl 2007; 13(11 Suppl 2): S48-S54.

14. Takada Y, Ueda M, Ito T, et al. Living donor liver transplantation as a second-line therapeutic strategy for patients with hepatocellular carcinoma. Liver Transpl 2006; 12: 912-919.

15. Lee SG, Hwang S, Moon DB, et al. Expanded indication criteria of living donor liver transplantation for hepatocellular carcinoma at one large-volume center. Liver Transpl 2008; 14: 935-945.

16. Ito T, Takada Y, Ueda M, et al. Expansion of selection criteria for patients with hepatocellular carcinoma in living donor liver transplantation. Liver Transpl 2007; 13: 1637-1644.

17. Soejima Y, Taketomi A, Yoshizumi T, et al. Extended indication for living donor liver transplantation in patients with hepatocellular carcinoma. Transplantation 2007; 83: 893-899. 


\section{Outcomes of LDLT and DDLT Recipients With HCC}

18. Sugawara Y, Tamura S, Makuuchi M. Living donor liver transplantation for hepatocellular carcinoma: Tokyo University series. Dig Dis 2007; 25: 310-312.

19. Bhangui $P$, Vibert $E$, Majno $P$, et al. Intention-to-treat analysis of liver transplantation for hepatocellular carcinoma: Living versus deceased donor transplantation. Hepatol 2011; 53: 1570-1579.

20. Otto G, Herber S, Heise M, et al. Response to transarterial chemoembolization as a biological selection criterion for liver transplantation in hepatocellular carcinoma. Liver Transpl 2006; 12 : 1260-1267.

21. Vitale A, D'Amico F, Frigo AC, et al. Response to therapy as a criterion for awarding priority to patients with hepatocellular carcinoma awaiting liver transplantation. Ann Surg Oncol 2010; 17 2290-2302

22. Cucchetti A, Cescon M, Bigonzi E, et al. Priority of candidates with hepatocellular carcinoma awaiting liver transplantation can be reduced after successful bridge therapy. Liver Transpl 2011; 17: 1344-1354.

23. Roberts JP, Venook A, Kerlan R, Yao F. Hepatocellular carcinoma: Ablate and wait versus rapid transplantation. Liver Transpl 2010; 16: 925-929.

24. Riaz A, Ryu RK, Kulik LM, et al. Alpha-fetoprotein response after locoregional therapy for hepatocellular carcinoma: Oncologic marker of radiologic response, progression, and survival. J Clin Oncol 2009; 27: 5734-5742.

25. Chan SL, Mo FK, Johnson PJ, et al. New utility of an old marker: Serial alpha-fetoprotein measurement in predicting radiologic response and survival of patients with hepatocellular carcinoma undergoing systemic chemotherapy. J Clin Oncol 2009; 27: 446-452.

26. Yao FY, Kerlan RK Jr., Hirose R, et al. Excellent outcome following down-staging of hepatocellular carcinoma prior to liver transplantation: an intention-to-treat analysis. Hepatol 2008; 48: 819-27.

27. MacDonald B, Sewell JL, Harper AM, Fix OK, Roberts JP, Yao FY; American Association for the Study of Liver Disease, Boston. Pre-transplant factors predicting tumor recurrence and mortality following liver transplantation for hepatocellular carcinoma: analysis of 1074 patients in UNOS region 5. [Abstract] Hepatol 2010; 52(Suppl): 371A.

28. Merani A, Majno P, Kneteman NM, et al. The impact of waiting list alpha-fetoprotein changes on the outcome of liver transplant for hepatocellular carcinoma. J Hepatol 2011; 55: 814-819.

29. Cillo U, Vitale A, Navaglia F, et al. Role of blood AFP mRNA and tumor grade in the preoperative prognostic evaluation of patients with hepatocellular carcinoma. World J Gastroenterol 2005; 11 $6920-6925$
30. Cillo U, Vitale A, Bassanello M, et al. Liver transplantation for the treatment of moderately or well-differentiated hepatocellular carcinoma. Ann Surg 2004; 239: 150-159.

31. DuBay D, Sandroussi C, Sandhu L, et al. Liver transplantation for advanced hepatocellular carcinoma using poor tumor differentiation on biopsy as an exclusion criterion. Ann Surg 2011; 253: 166-172.

32. Berg CL, Merion RM, Shearon TH, et al. Liver transplant recipient survival benefit with living donation in the MELD allocation era. Hepatol 2011; 54: 1313-1321.

33. Sandhu L, Sandroussi C, Guba M, et al. Living Donor Liver Transplantation Versus Deceased Donor Liver Transplantation for Hepatocellular Carcinoma: Comparable Survival and Recurrence. Liver Transpl 2012; 18: 315-322.

\section{Supporting Information}

Additional Supporting Information may be found in the online version of this article.

Table S1: Characteristics of the 126 MELD era HCC patients at donor evaluation.

Table S2: Characteristics of the 126 MELD era HCC transplanted patients at transplant.

Table S3A: Single-variable Cox models for HCC recurrence.

Table S3B: Single-variable Cox models for death.

Figure S1: Characteristics of HCC patients among those who had LDLT and recurred within 1 year posttransplant, all other LDLTs and DDLTs. Tumor grade was available for $11(79 \%), 57(66 \%)$ and $62(64 \%)$, in the three respective groups.

Please note: Wiley-Blackwell is not responsible for the content or functionality of any supporting materials supplied by the authors. Any queries (other than missing material) should be directed to the corresponding author for the article. 